\title{
De la sobrevida al desarrollo integral de la infancia: Pasos en el desarrollo del sistema de protección integral a la infancia
}

\author{
HELIA MOLINA M. ${ }^{1}$, MIGUEL CORDERO V. ${ }^{2}$, VERÓNICA SILVA V. ${ }^{3}$ \\ 1. Jefe de División de Políticas Públicas Saludables y Promoción, Ministerio de Salud de Chile. \\ 2. Sistema de Protección Integral a la Infancia - Chile Crece Contigo, Ministerio de Salud de Chile. \\ 3. Secretaria Ejecutiva Sistema de Protección Social, Ministerio de Planificación de Chile.
}

Palabras clave: Sobrevida, desarrollo, protección, infancia.

\section{Introducción}

Anualmente más de 200 millones de niños y niñas menores de 5 años de países en desarrollo no logran desplegar su optimo potencial de desarrollo físico, psíquico y social (GranthamMcGregor et al. 2007). Los factores que inciden sobre estos resultados son múltiples y responden tanto a condiciones biológicas, como sociales y ambientales, dístales y proximales al niño que han sido sistematizadas en base al modelo ecológico del desarrollo humano (Bronfenbrenner 1979).

El modelo ecológico del desarrollo infantil, considera que el desarrollo en su conjunto depende de la interacción dinámica del niño o niña con su entorno. En esta visión (figura 1), los ambientes y entornos están representados por el Estado, la comunidad y la familia, y cada uno de ellos posee sus normas y valores; el Estado a través de formulación de políticas y marcos institucionales; la comunidad, mediante sus modelos de organización y participación; y las familias, en su papel trascendente de protección, cuidado y satisfacción de necesidades inmediatas de la niñez.
El modelo ecológico aplicado al desarrollo reconoce los determinantes biológicos asociados a la genética y a situaciones de salud, sin embargo resalta la evidencia acumulada, proveniente de las neurociencias, ciencias del comportamiento, ciencias sociales y económicas que muestran como factor determinante del desarrollo humano, a aquellos factores a asociados a las condiciones de vida, educación de los padres, entorno físico y psicosocial, en donde el niño nace, crece, juega, se educa, así como la forma en que se accede a servicios de salud, educación, protección social y las formas de organización de las comunidades (Benguigui Y., Molina H. Editores 2004).

Así mismo, los últimos 50 años de investigación neurobiológica ha probado la existencia de períodos críticos o sensibles en el desarrollo temprano de varios sistemas (visual, lenguaje), que definen tiempos y ventanas de oportunidad en las que se "imprimen o marcan" en el desarrollo cerebral, las posibilidades para desplegar una función, con múltiples efectos a corto y largo plazo en el desarrollo cognitivo, psicológico y social que impactan de forma permanente en la salud y el desarrollo a lo largo de toda la vida.

Desde las ciencias económicas hay sólida evidencia de que una fuerte inversión social en

Correspondencia a:

Helia Molina M.

E-mail: hmolina@minsal.cl 


\section{La familia, la comunidad y las políticas públicas como entorno del desarrollo infantil}

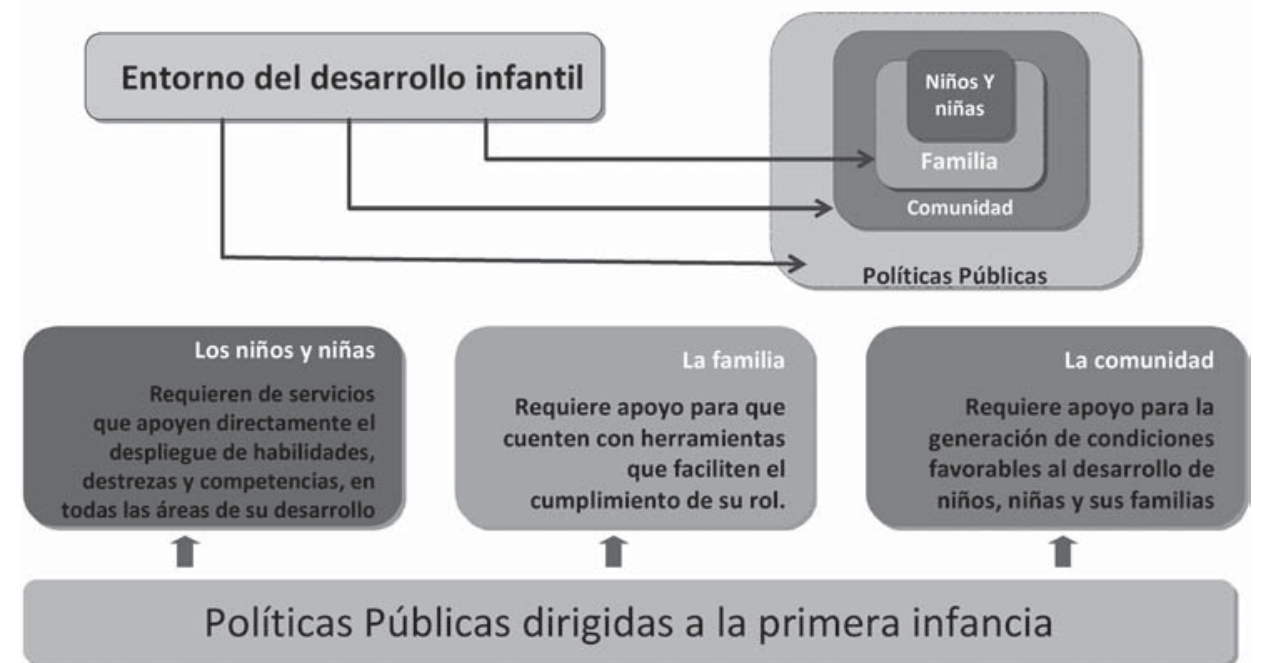

Figura 1. Modelo Ecológico.

primera infancia tiene el poder de romper el ciclo intergeneracional de la pobreza (Sen A. 1999) y que la tasa de retorno de la inversión en programas y políticas publicas para primeros 6 años de vida puede ser hasta de 8 veces, retorno que va disminuyendo progresivamente al aumentar la edad (Carnero P. \& Heckman J. 2003).

Recientemente Organización Mundial de la Salud, reconociendo el Desarrollo infantil temprano como un determinante de la salud y el desarrollo humano, lo incorporo como una de las áreas nucleares a trabajar en las redes de conocimiento generadas por la Comisión Mundial de Determinantes Sociales (2005). El objetivo de esta comisión fue recabar datos científicos sobre posibles medidas e intervenciones dirigidas a reducir las inequidades sanitarias susceptibles de solución dentro de cada país y entre los países.

El informe final de la red de conocimientos sobre desarrollo infantil temprano muestra que las intervenciones en la primera infancia pueden actuar como un poderoso equalizador de las inequidades en salud y se hacen importantes recomendaciones para los países. Mucho de estas intervenciones son de bajo costo y de aplicación inmediata.
En sus recomendaciones generales la comisión (2008) afirma que los países deben:

1. Comprometerse con una estrategia integral que aborde los primeros años de vida y ponerla en marcha, partiendo de los programas de supervivencia infantil existentes y ampliando el alcance de las intervenciones destinadas a la primera infancia para que incluyan el desarrollo socioemocional y lingüistico-cognitivo.

2. Establecer un mecanismo interinstitucional que garantice la coherencia de las políticas para el desarrollo de la primera infancia, a fin de que el conjunto de las instituciones apliquen un enfoque integral.

3. Velar por que todos los niños, madres y otras personas que tengan niños a su cargo se beneficien de un amplio conjunto de programas y servicios de calidad para el desarrollo de la primera infancia, con independencia de su capacidad de pago.

\section{EI Desarrollo Integral de la Infancia en Chile: Una deuda pendiente}

En América latina y muy especialmente en los países del cono sur en los últimos años, se 
ha producido un significativo y sostenido descenso de la mortalidad infantil. En particular Chile ha alcanzado una mortalidad de 7.9 por mil nacidos vivos en el año 2005, según el último informe de Los Objetivos de Desarrollo del Milenio. Estos datos y otros son muy semejantes a los países desarrollados con ingresos per cápita hasta 10 veces mayor, lo que demuestra la eficacia y eficiencia de las políticas, planes y programas sociales y de salud materno infantil desarrollados en Chile en las décadas anteriores.

Sin duda han sido factores claves políticas públicas sostenidas que han permitido el avance en saneamiento básico, agua potable, acceso a servicios de salud, atención profesional del parto y los programas de inmunización.

Pese a los indiscutibles avances en estos indicadores de salud el desarrollo integral y la calidad de vida en la infancia sigue siendo una deuda pendiente para Chile. Datos recientes

Tabla 1. Prevalencia de Rezago según edad. Menores de 5 años

\begin{tabular}{lccc}
\hline & n & $\begin{array}{c}\text { Prevalencia } \\
\text { \% }\end{array}$ & $\begin{array}{c}\text { IC 95 } \\
\text { \% }\end{array}$ \\
\hline 2-11 meses & 55205 & 24,9 & $18,3-31,5$ \\
1 año-1 año 11 meses & 68551 & 28,0 & $22,0-33,9$ \\
2 años-2 años 11 meses & 75630 & 28,4 & $22,2-34,7$ \\
3 años-3 años 11 meses & 87261 & 34,2 & $27,5-41,0$ \\
4 años-4 años 11 meses & 87523 & 33,4 & $27,0-39,8$ \\
Total & 374170 & 29,9 & $27,1-32,8$ \\
\hline
\end{tabular}

Fuente: Informe Final II Encuesta Nacional de Calidad de Vida y Salud 2006. División de Planificación Sanitaria. Ministerio de Salud. del modulo infantil en la Encuesta Nacional de Calidad de Vida (2006), realizada a 4.997 hogares con niños o niñas menores de 5 años, con representatividad nacional, regional y urbanorural, muestra que cerca de un $30 \%$ de niños y niñas chilenos (tabla 1), no son capaces de realizar todas las funciones que están en proceso de desarrollo para su grupo de edad, ubicándolos en situación de riesgo de potencial retraso en el desarrollo. Cuando estos datos se analizan considerando los quintiles de ingreso familiar, los resultados muestran importantes brechas de inequidad dentro del primer y cuarto año de vida especialmente (tabla 2). En tanto que las prevalencias de retraso en los quintiles de ingreso extremos muestran notables diferencias en todos los rangos etareos (figura 2).

\section{Chile Crece Contigo: Un sistema integrado de servicios a favor de la primera infancia}

A un mes de iniciado su gobierno, por decreto supremo (Decreto Supremo n ${ }^{\circ} 072$, 4 abril de 2006) la Presidenta de la República Michelle Bachelet constituyó un Consejo Asesor Presidencial para la Reformas de las Políticas de Infancias. Tal consejo tuvo por mandato "Elaborar un diagnostico de la situación actual y de las insuficiencias existentes en materia de protección a la infancia, para luego, formular y proponer un conjunto de políticas y medidas idóneas para efectos de implementar un sistema de protección a la infancia".El organismo entregó a la Mandataria un informe denomina-

Tabla 2. Prevalencia total (\%) con IC $95 \%$ de Rezago según Quintil de ingreso Menores de 5 años

\begin{tabular}{lccccc}
\hline & $\begin{array}{c}\mathbf{1}^{\mathbf{0}} \\
\text { Prev IC 95\% }\end{array}$ & $\begin{array}{c}\mathbf{2}^{\mathbf{0}} \\
\text { Prev IC 95\% }\end{array}$ & $\begin{array}{c}\mathbf{3}^{\mathbf{0}} \\
\text { Prev IC 95\% }\end{array}$ & $\begin{array}{c}\mathbf{4}^{\mathbf{0}} \\
\text { Prev IC 95\% }\end{array}$ & $\begin{array}{c}\mathbf{5}^{\mathbf{0}} \\
\text { Prev IC 95\% }\end{array}$ \\
\hline 2-11 meses & $31.015 .4-46,5$ & $19.77 .7-31,8$ & $33.518 .2-48,8$ & $24.59 .8-39,1$ & $14.42 .7-26,1$ \\
1 año - 1 año 11 meses & $35.622 .4-48,8$ & $30.416 .9-43,9$ & $31.317 .9-44,6$ & $19.58 .8-30,1$ & $22.57 .3-37 ., 8$ \\
2 años - 2 años 11 meses & $37.020 .7-53,2$ & $30.017 .3-42,7$ & $39.524 .7-54,3$ & $15.14 .7-25,6$ & $21.88 .2-35,5$ \\
3 años - 3 años 11 meses & $34.020 .4-47,7$ & $42.524 .4-60,6$ & $27.014 .7-39,3$ & $32.218 .9-45,4$ & $39.822 .4-57,2$ \\
4 años - 4 años 11 meses & $39.726 .4-53,0$ & $35.922 .5-49,4$ & $43.928 .6-59,3$ & $29.815 .0-44,7$ & $14.92 .3-27,5$ \\
Total & $35.929 .5-42,4$ & $31.625 .2-38,0$ & $34.928 .5-41,3$ & $24.518 .5-30,4$ & $23.116 .4-29,9$ \\
\hline
\end{tabular}

Fuente: Informe Final II Encuesta Nacional de Calidad de Vida y Salud 2006. División de Planificación Sanitaria. Ministerio de Salud. 


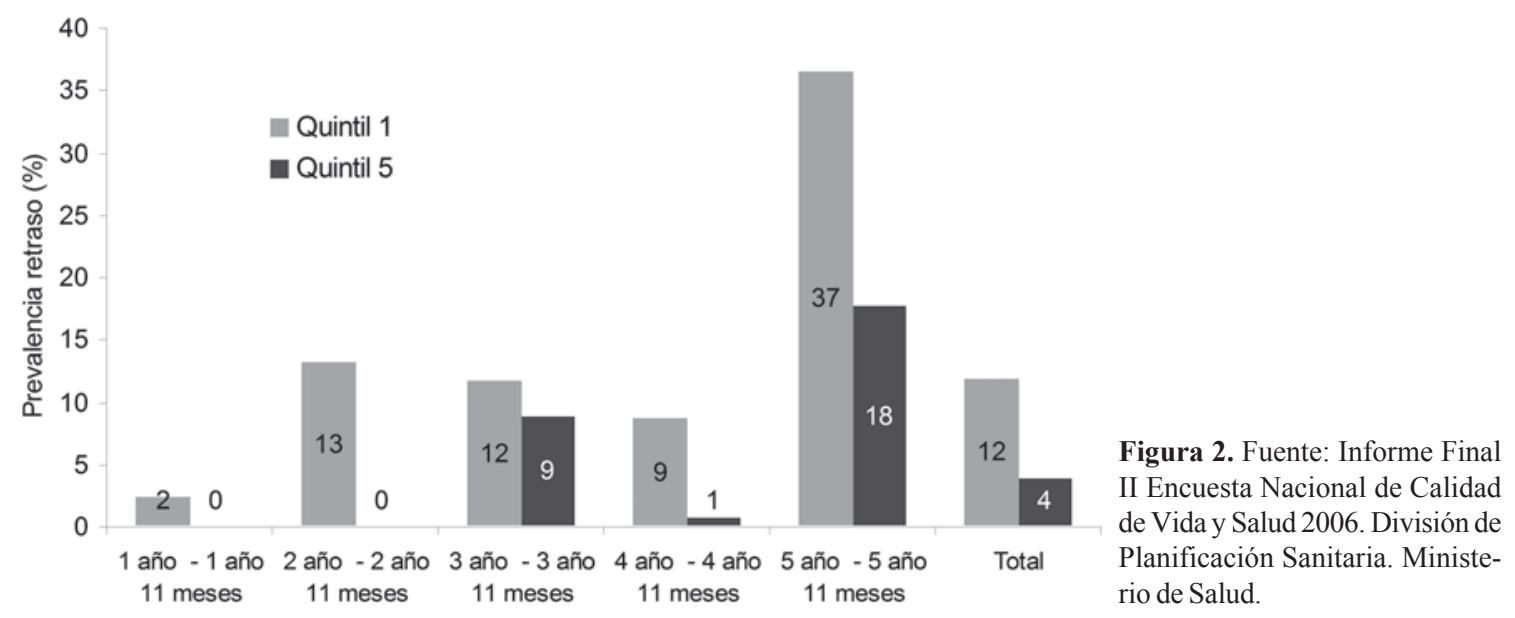

do "El Futuro de los Niños es Siempre Hoy". Tal informe contenía las propuestas de consenso para implementar una reforma profunda de los servicios sociales y de salud para la primera infancia. Tal propuesta se baso en un amplio proceso de participación ciudadana, donde se escuchó, analizó e incorporó las visiones de especialistas, organizaciones de la sociedad civil y de la ciudadanía en general; se recibieron a más de 45 grupos y organizaciones, además de realizar audiencias en cada región, además se recibieron más de 17 mil correos electrónicos de adultos y más de 7 mil de niños(as) de todo el país.

El Informe del Consejo fue recibido para su análisis por un Comité de Ministros de Infancia, coordinado por el Ministerio de Planificación. El objetivo central de este comité técnico fue analizar las propuestas del Consejo Asesor Presidencial, estudiar su viabilidad, y proponer iniciativas de implementación. Finalmente, parte importante de las recomendaciones contenidas en el informe final del consejo llegaron a plasmarse en lo que hoy se denomina Sistema de Protección Integral a la Infancia Chile Crece Contigo (ChCC).

Este sistema ha sido creado con la misión de igualar oportunidades de desarrollo en niños, niñas, sus familias y comunidades. El sistema busca generar una red integrada de servicios dirigidos a optimizar el ambiente de desarrollo desde la gestación y hasta los cuatro años de edad, edad en la cual se espera que muchos niños y niñas de esta generación ingresen al sistema de educación preescolar.

La operación de este sistema se sustenta en la gestión local de los servicios (figura 3) con múltiples apoyos desde el gobierno central. Así cada repartición pública pone a disposición del sistema su oferta programática en materia de primera infancia en un territorio particular. Por ejemplo, el sector salud integra sus múltiples acciones y servicios dirigidos a familias con niños y niñas menores de 4 años. Por otra parte, el sector de educación, comienza un progresivo incremento en la oferta de servicios de apoyo al cuidado infantil y de ecuación temprana.

Es interesante notar que una de las principales recomendaciones del consejo para el sector salud, fue hacer una profunda modificación del enfoque en la atención de la salud de los niños, niñas y sus familias. Ampliar la visión biomédica clásica del crecimiento y desarrollo hacia un modelo con mayor énfasis en la dimensión psicosocial, que considere centralmente la dimensión social del desarrollo infantil.

\section{Chile Crece Contigo es una intervención universal y selectiva}

Chile Crece Contigo se erige como una Red que busca la articulación e integración de los servicios a favor del desarrollo infantil con el objeto de responder oportuna y pertinentemente a las necesidades de cada niño, niña y familia (figura 4) .

En esta red los servicios de salud son funda- 


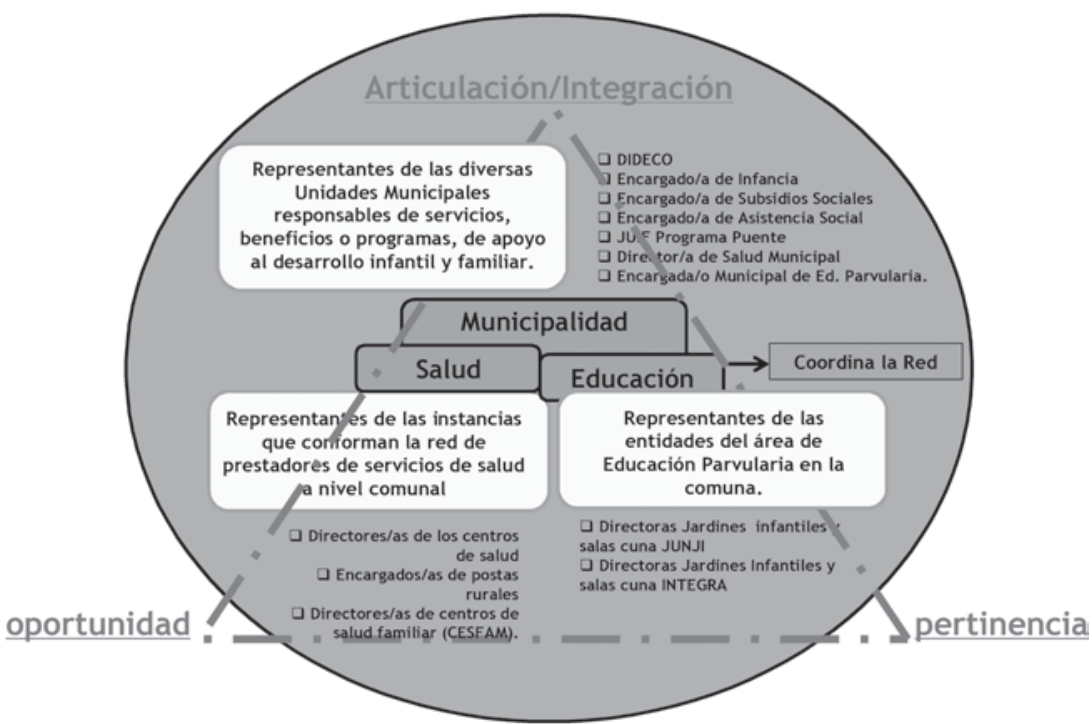

La Red Comunal es donde se plasma Chile Crece Contigo en cada territorio

Figura 3.

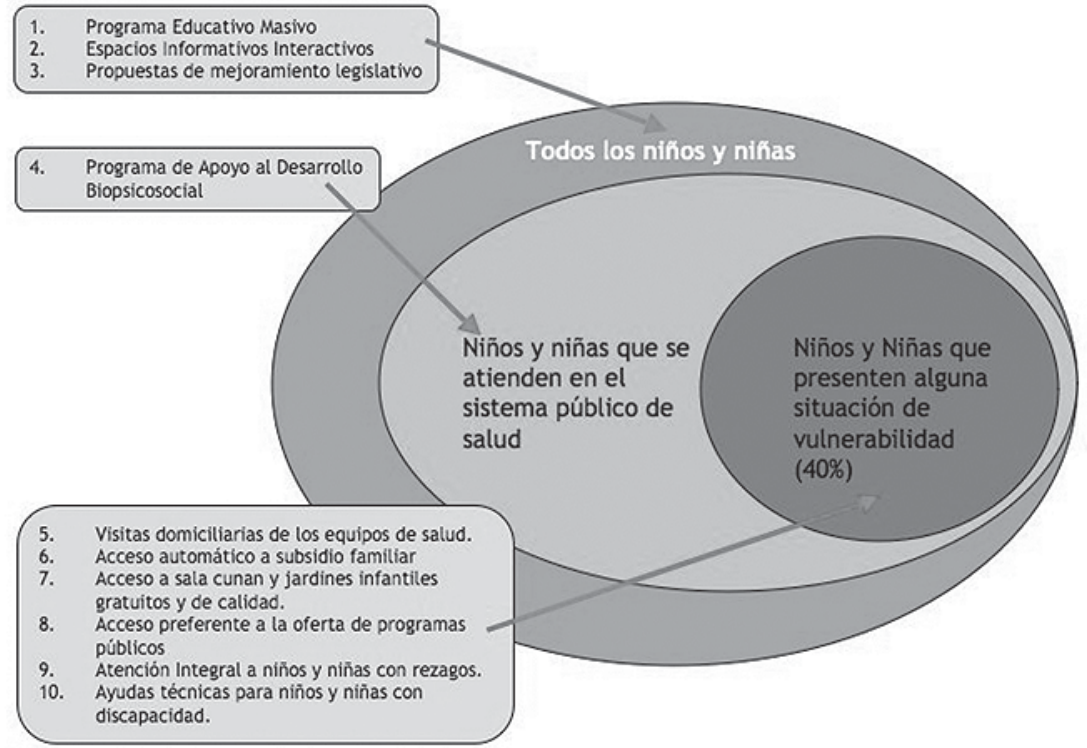

Figura 4.

mentales para el sistema, dado que es mediante los frecuentes contactos del niño o niña y su familia se puede dar un seguimiento cercano a la trayectoria de desarrollo. En Chile el 84\% de los niños y niñas nacen el sistema público, por ello este constituye la puerta de entrada privilegiada a un seguimiento personalizado del desarrollo de cada niño, niña y su familia.

La instalación progresiva de importantes modificaciones a los programas dirigidos a la salud infantil y a la salud sexual y reproductiva, es la base de lo que se denomina componente de Salud del Sistema o "Programa de Apoyo al Desarrollo Biopsicoscial" (PADBP) que se implementa en los servicios de salud de la atención primaria, secundaria y terciario.

Así, el PADBP sirve de plataforma para la activación de alarmas e intervenciones selecti- 
vas de otros servicios, para aquellos niños o niñas y sus familias que viven alguna situación de vulnerabilidad o que pertenecen al $40 \%$ más de las familias chilenas.

Las acciones contempladas en el PADBP incluyen algunas que históricamente el sector salud ha realizado en el proceso de atención de la gestación y los primeros 4 años de vida, sin embargo el enfoque y rediseño de gran parte de tales acciones implica un salto cualitativo en la calidad de los servicios que se ofrece.

El PADBP considera acciones en los tres niveles de la red mediante las siguientes estrategias:

1. Fortalecimiento de los Cuidados prenatales.

2. Atención personalizada del proceso de nacimiento.

3. Atención integral durante la hospitalización de una mujer en gestación o de un niño/a.

4. Fortalecimiento del control de salud infantil.

5. Fortalecimiento de las intervenciones en niños y niñas en riesgo o con rezagos en su desarrollo.

El fortalecimiento del control prenatal para todas las usuarias del sistema publico de salud incluye entre otras actividades la entrega de una guía anticipatoria denominada "Empezando a Crecer", la inclusión de una evaluación psicosocial abreviada como sistema de vigilancia y tamizaje para la activación de prestaciones diferenciadas (visita domiciliaria integral por ejemplo), oferta universal de educación prenatal y la garantía de acompañamiento de una figura significativa en todo el proceso.

El componente prenatal puede resultar en la reducción de la tasa de cesáreas, reducción de los niveles de ansiedad al parto, reducción de la incidencia de exposición prenatal a tabaco, alcohol y otras drogas de abuso, reducción de la violencia de género y mayor empoderamiento de las mujeres mediante la educación sobre sus derechos.

Se establecen mecanismos efectivos de coordinación entre el nivel terciario y la atención primaria con el objeto de dar una efectiva continuidad en la atención entre el centro hospitalario y el centro de atención primaria más cercano al lugar de residencia de esa familia.

Las intervenciones posteriores al nacimiento incluyen la entrega de una guía anticipatoria del desarrollo entre los 0 y 24 meses denominada "Acompañándote a Descubrir", materiales para la estimulación del desarrollo del lenguaje y del juego, y el aumento progresivo en la oferta de programas educativos dirigidos apoyar el proceso de desarrollo en todas las familias.

La oferta integrada de múltiples intervenciones busca reducir la brecha de rezagos en el desarrollo (motor, cognitivo, social o emocional) infantil entre grupos socioeconómicos extremos. La mayor parte de estas intervenciones se producen en el nivel de atención primaria y están dirigidas a la promoción del desarrollo infantil saludable y la prevención mediante la educación grupal para el desarrollo de habilidades parentales, visitas domiciliarias integrales y acciones de coordinación y trabajo integrado entre los centros de salud, sala cunas y jardines infantiles de un territorio. Por otra parte se considera como prioritario la integración de niños y niñas con mayor vulnerabilidad (biológica o social) a alguna modalidad estimulación temprana, sea esta por la vía de la educación temprana o por modalidades no convencionales, tale como las salas de estimulación en los centro de salud, o modalidades itinerantes de estimulación del desarrollo en territorios con una mayor dispersión geográfica.

Pese a lo central que puede parecer el componente de salud de este sistema, se debe considerar que para que Chile Crece Contigo se encuentre operando en un territorio determinado, debe existir una red más amplia de servicios en coordinación que incluyen la educación preescolar y otras múltiples agencias del gobierno que operan programas que apoyan el desarrollo de niños, niñas, sus familias y comunidades. Esta red red comunal básica (figura 3), activa y conecta los múltiples servicios requeridos disponibles en el territorio. Mucho de la evidencia disponible actualmente sobre la efectividad de programas como $\mathrm{ChCC}$, muestra que es esta red la que puede hacer la diferencia.

La oferta integrada de servicios se basa en el desarrollo de las redes comunales de $\mathrm{ChCC}$, con el objeto de lograr un acceso oportuno y pertinente a diferentes modalidades de estimulación del desarrollo acordes a las necesidades particulares de las familias y comunidades.

Es por ello que se debe considerar que Chile 
Crece Contigo, más que la entrega de un servicio o prestación específico, es un esfuerzo nacional por mejorar los servicios ya existentes mediante el rediseño, fortalecimiento y gestión territorial para el logro de un desarrollo integral de la primera infancia.

\section{Reflexiones y recomendaciones para el proceso}

El proceso de instalación de Chile Crece Contigo es reciente y por lo tanto es prematuro evaluarlo. Las primeras 161 comunas comenzaron la instalación en Agosto del año 2007 y recién en el segundo trimestres de 2008 han comenzado nacer los primeros niños y niñas afectados por la intervención del sistema desde su gestación. Así mismo, datos provenientes de otros programas con objetivos similares (Sure Start 2008) muestran que muchos programas locales no están totalmente operativos sino hasta 3 años de iniciada su implementación.

Los diagnósticos están hechos, tenemos la evidencia científica que avala estas intervenciones, existe decisión política para implementar las medidas necesarias. Esta tarea requiere complejas coordinaciones, que involucrarán necesariamente del desarrollo de nuevos mecanismos de gestión, mayores niveles de empoderamiento y liderazgo local.

La evaluación del proceso e impacto de Chile Crece Contigo podrá enseñarnos mucho sobre cómo mejorar las condiciones del ambiente de desarrollo de muchos niños y niñas. Los datos del proceso de instalación son prometedores en múltiples sentidos; $\mathrm{ChCC}$ ha generado una gran convocatoria en todos los servicios públicos, imprimiendo una nueva mística al trabajo, así mismo los datos crudos muestran un crecimiento exponencial de prestaciones de salud relegadas al olvido por el modelo curativo, tales como la visita domiciliaria y los espacios educativos para la promoción del desarrollo infantil y la crianza.

Sabemos que el desafío es grande, la instalación de Chile Crece Contigo es una de las mayores tareas en materia de gestión de servicios públicos integrados, por lo mismo su agenda de trabajo requiere del apoyo político y de un presupuesto seguro para que todas las reparticiones públicas relacionadas al sistema puedan cumplir sus compromisos.

Chile Crece Contigo no es sólo una agenda programatica de un gobierno, es un desafío nacional y un compromiso ético. En palabras de Gabriela Mostral "El Futuro de los Niños es Siempre Hoy", ellos no pueden esperar.

\section{Referencias}

1.- Benguigui Y, Molina H: Editores (2004) Encuentro Internacional de Desarrollo Infnatil en el marco de loa Objetivos de Desarrollo del Mileno. Belém do Pará, Brasil, 8 al 10 de junio de 2004. Organización Panamericana de la Salud.

2.- Bronfenbrenner $U$ : The ecology of human development. Cambridge: Harvard University Press (existe edición en castellano en La ecología del desarrollo humano, Barcelona: Paidós, 1987). 1979.

3.- Carneiro P, Heckman JJ: Human capital policy. In J. J. Heckman, A. B. Krueger, and B. M. Friedman (Eds.), Inequality in America: What Role for Human Capital Policies? Cambridge, MA: MIT Press. 2003.

4.- Consejo Presidencial Infancia. El futuro de los niños es siempre hoy. Propuestas del Consejo Asesor Presidencial para la Reforma de las Políticas de Infancia. Junio. 2006.

5.- Grantham-McGregor S, Cheung Y B, Cueto S, Glewwe $P$, Richter L, Strupp B, and the International Child Development Steering Group: Child development in developing countries 1 . Developmental potential in the fi rst 5 years for children in developing countries. Lancet 2007; 369: 60-70.

6.- Jiménez J, Romero MI: Reducing Infant Mortality In Chile: Success In Two Phases. Health Affairs 2007; 26 (2): 458-65.

7.- Irwin L, Siddiqi A, Hertzman C: Early Chile Development: A Powerful Equalizer. Final Report for the World Health Organization's. Commission on the Social Determinants of Health. 2007.

8.- Sen AK, Brundtland GH: Conferencias Magistrales: Romper el ciclo de la pobreza Invertir en la infancia. Banco Interamericano de Desarrollo. 1999.

9.- Ted Melhuish, NESS Team: Findings from the National Evaluation of Sure Start (NESS). Sure Satart National Conference. 2008. 